\title{
Probability distributions of the electroencephalogram envelope of preterm infants
}

\author{
Ryoya Saji* \\ Brain Science Institute, Tamagawa University, Tokyo, Japan \\ Kyoko Hirasawa \\ Tokyo Women's Medical University Hospital, Tokyo, Japan \\ Masako Ito \\ Tokyo Women's Medical University Hospital, Tokyo, Japan \\ Satoshi Kusuda \\ Tokyo Women's Medical University Hospital, Tokyo, Japan \\ Yukuo Konishi \\ Center for Baby Science, Doshisha University, Kyoto, Japan \\ Gentaro Taga \\ Graduate School of Education, The University of Tokyo, Tokyo, Japan
}

\begin{abstract}
Objective: To determine the stationary characteristics of electroencephalogram (EEG) envelopes for prematurely born (preterm) infants and investigate the intrinsic characteristics of early brain development in preterm infants.

Methods: Twenty neurologically normal sets of EEGs recorded in infants with a post-conceptional age (PCA) range of 26-44 weeks (mean 37.5 \pm 5.0 weeks) were analyzed. Hilbert transform was applied to extract the envelope. We determined the suitable probability distribution of the envelope and performed a statistical analysis.

Results: It was found that (i) the probability distributions for preterm EEG envelopes were best fitted by lognormal distributions at 38 weeks PCA or less, and by gamma distributions at 44 weeks PCA; (ii) the scale parameter of the lognormal distribution had positive correlations with PCA as well as a strong negative correlation with the percentage of low-voltage activity; (iii) the shape
\end{abstract}

${ }^{*}$ Corresponding author at Brain Science Institute, Tamagawa University, 6-1-1 TamagawaGakuen, Tokyo, 1948610, Tel. +81 42739 8680, Fax. +81 42739 8666. E-mail address: ryosaji@tamagawa.ac.jp (R.Saji).

Preprint submitted to Elsevier

August 26, 2014

(C) 2014. This manuscript version is made available under the Elsevier user license http://www.elsevier.com/open-access/userlicense/1.0/ 
parameter of the lognormal distribution had significant positive correlations with PCA; (iv) the statistics of mode showed significant linear relationships with PCA, and, therefore, it was considered a useful index in PCA prediction.

Conclusion: These statistics, including the scale parameter of the lognormal distribution and the skewness and mode derived from a suitable probability distribution, may be good indexes for estimating stationary nature in developing brain activity in preterm infants.

Significance: The stationary characteristics, such as discontinuity, asymmetry, and unimodality, of preterm EEGs are well indicated by the statistics estimated from the probability distribution of the preterm EEG envelopes.

Keywords: probability distribution, electroencephalogram (EEG), envelope, prematurely born (preterm) infant, statistical mathematical analysis, brain maturation 


\section{Introduction}

Electroencephalograms (EEGs) for prematurely born (preterm) infants usually show high-voltage amplitudes of over $300 \mu \mathrm{V}$ and are discontinuous, with long periods of quiescence. That is, EEGs display in bursts that are primarily comprised of high-amplitude delta waves alternating with low-voltage intervals, and this pattern shortens as the gestational age of the infant increases (Anderson et al., 1985; Selton et al., 2000; Clancy et al., 2003; Vanhatalo et al.,2005). Furthermore, specific waveforms and patterns, such as the beta-delta complex (Dreyfus-Brisac, 1964), temporal theta and alpha bursts (Hughes et al., 1983), and frontal sharp waves (Kellaway and Crawley, 1964), are observed as gestational age increases (c.f. see review Scher, 2008). While spectral analyses that are based on fast Fourier transforms have been extensively applied to assess the frequency oscillations in preterm EEGs over several decades (Parmelee et al., 1967; Willekens et al., 1984), a simple visual inspection provides a practical advantage in terms of the day-to-day interpretation of preterm EEGs. However, except for experienced electroencephalographers, it is generally difficult for medical personnel to assess the continually changing EEG patterns, which hinders the accurate interpretation of the subtle differences in preterm EEGs.

The wave envelope is widely utilized in the statistical analysis of complex and nonstationary signals, such as those of mechanical vibrations (Sheen, 2010), physiological acoustic signals (Sovijarvi et al., 2000), cardiac sound signals (Liang et al., 1997), and the amplitudes of ocean waves (Tayfun and Lo, 1990). Because the envelope generally changes at a slower rate compared to the signal's temporal variations, its characteristics provide a direct measure of the instantaneous wave amplitude. The concept of the wave envelope was first introduced by Rice (1945). Subsequently, some methods of envelope extraction from nonstationary time series have been suggested (Langley, 1986). Currently, the most commonly used extraction method utilizes the Hilbert transform. The statistical characteristics of wave envelopes have been described by various probability distributions. It is known that the most popular distribution for envelopes that are extracted from EEG is the Rayleigh distribution (Saunders, 1963). The Rayleigh is always unimodal as it has a single peak and it is skewed to the right. In addition, the Rayleigh fits well in the envelope process, which is described as a narrow-band Gaussian random (NBGR) process (Dick and Vaughn, 1970). Although preterm EEGs would not completely and correctly describe as the NBGR, the envelope brings us effective information about the amplitude. Because of the complex and nonstationary nature mainly confined in the frequency of $\delta$-band, examination of the envelope and clarification of the statistical properties in the envelope not only offer objective validity and provide reasonably accurate information for visualizing preterm EEG patterns, but also aid in investigating the intrinsic characteristics of early human brain development.

The aims of this study were to determine the stationary characteristics of preterm EEG envelopes and investigate the intrinsic characteristics of early brain development in humans. We focused on the features of discontinuity, asymmetry, and unimodality. To determine these characteristics, we extracted 
the envelope from preterm EEG signals with the Hilbert transform and then identified a probability distribution that was suitable to fit the envelopes. To obtain a better understanding of early human brain development, we compared three popular right-skewed and unimodal distributions: the Rayleigh, lognormal, and gamma. Subsequently, applying the maximum likelihood method, we identified the maximum likelihood model. Namely, the most suitable distributions were identified among the three probabilities. We then estimated the statistics derived from the probability distribution and examined the typical characteristics between the intrinsic mechanism and stationary nature of preterm EEG. Finally, we discuss the advantages of our proposed method in terms of clinical evaluations of brain maturation.

\section{Methods}

\subsection{Subjects}

Twelve clinically stable preterm infants (7 females) at the Tokyo Women's Medical University Hospital were chosen as the subjects for our study. The mean gestational age \pm standard deviation at birth was $32.0 \pm 4.5$ weeks (range: 25 weeks and 3 days to 36 weeks and 6 days), and the mean birth weight was $1,680 \pm 919 \mathrm{~g}$ (range: $558 \mathrm{~g}$ to $3,028 \mathrm{~g}$ ). All infants were considered low risk, because no signs of postnatal physical and neurological disease were observed. Moreover, the Apgar scores at 5 min for all infants were 7 to 10 (mean, $8.6 \pm$ 0.9 ), indicating a relatively low score for the risk of neonatal death (Casey et al., 2001). This study was approved by the Tokyo Women's Medical University

Hospital review board, and informed consent was obtained from the parents of each of the subjects.

\subsection{EEG recordings}

Twenty sets of EEGs were obtained at the mean post-conceptional age (PCA: gestational age + postnatal age) of $37.0 \pm 5.0$ weeks (range: 26 weeks and 4 days to 44 weeks and 5 days). All EEGs were recorded at the infants' bedside with six surface $\mathrm{Ag}-\mathrm{AgCl}$ electrodes (Fp1, Fp2, C3, C4, O1, and O2) and two reference electrodes that were affixed to the ears (A1 and A2) with paste, according to the International 10-20 system. Impedance values were maintained at less than 10 $\mathrm{k} \Omega$ during the recording, and the sampling frequency was set at $500 \mathrm{~Hz}$. We used a high-pass filter of $0.5 \mathrm{~Hz}$ and a high-cut filter of $120 \mathrm{~Hz}$ along with a notch filter of $50 \mathrm{~Hz}$. All recordings were obtained with a digital EEG instrument (EEG-9100; Nihon Kohden Corporation, Tokyo, Japan). Thus, we analyzed EEGs $(20$ sets $\times$ six leads; Fp1-A2, Fp2-A1, C3-A2, C4-A1, O1-A2, and O2A1) that were recorded over an average duration of $45.8 \pm 19.8$ min (range: 15.8 min to $80.0 \mathrm{~min}$ ) after eliminating epochs in which body movement and/or electromyographic activity were observed on visual inspection. 


\subsection{Envelope extraction}

The envelope of the analyzed signal can be provided by the well-known Hilbert transform (Middleton, 1960), which is denoted by $\mathrm{H}(\cdot)$. To summarize the key idea behind the Hilbert transform, let us denote an analyzed signal $x(t)$ as a real-valued signal. Its Hilbert transform is defined as

$$
H(x(t))=x(t) * \frac{1}{\pi t}=\frac{1}{\pi} \int_{-\infty}^{\infty} \frac{x(\tau)}{t-\tau} d \tau .
$$

Here, the principal value of the integral is used, and the ${ }^{*}$ indicates the convolution operator. A basic characteristic of the Hilbert transform is that it shifts the phase of the signal by $90^{\circ}$; i.e., $H(H(x(t)))=-x(t)$. Furthermore, a complex analytic signal $z(t)$ can be expressed as

$$
z(t)=x(t)+i H(x(t))=E(t) \exp i \phi(t) .
$$

Here $E(t)=\sqrt{x(t)^{2}+H(x(t))^{2}}$ and $\phi(t)=\arctan (H(x(t)) / x(t)) . \quad E(t)$ is a mathematical variable that contain information about the amplitude of $x(t)$. In this paper, the $E(t)$ that is calculated with equations (1) and (2) is called the envelope of $x(t)$.

\subsection{Quantitative analysis of discontinuous patterns}

Many previous studies have described the discontinuity in preterm EEGs (Anderson et al., 1985; Connel et al., 1987; Eyre et al., 1988; Goto et al., 1992). The definition of low-voltage activity as a discontinuity differs according to the individual study. The commonly used definition is a waveform with an amplitude of $15-30 \mu \mathrm{V}$ lasting for more than $2-5$ seconds. We determined lowamplitude activity on the basis of Hayakawa et al.'s (2001) definition: segments for which the amplitude of the envelope $E(t)$ was $\leq 30 \mu \mathrm{V}$ and that continued for $>5 \mathrm{~s}$ were defined as low-amplitude activity. For the $E(t)$, we calculated (i) the proportion (percentage: \%) accounted for by low-amplitude activity of the entire length, (ii) the maximum length of low-amplitude activity segments (in seconds), and (iii) the mean length of low-amplitude activity segments (in seconds), respectively. Then we investigated these properties as indicators of discontinuity.

\subsection{Identification of probability distribution}

Let $x(t)$ and $H(x(t))$ be independent random variables with $\sim N\left(0, \theta^{2}\right)$, and, then, the envelope $E(t)$ follows the Rayleigh distribution:

$$
f(x ; \theta)=\frac{x}{\theta^{2}} \exp \left(-\frac{x^{2}}{2 \theta^{2}}\right), x>0 .
$$

The Rayleigh involves only the scale parameter $\theta>0$ and becomes a candidate for the probability distribution of EEG envelope. The Rayleigh always has a single peak and is skewed to the right. This indicates that unimodality and 
positive skewness are considered common characteristics among probability distributions. Other continuous probability distributions with these characteristics include the lognormal distribution,

$$
f(x ; \mu, \sigma)=\frac{1}{\sqrt{2 \pi \sigma^{2}} x} \exp \left(-\frac{(\ln (x)-\mu)^{2}}{2 \sigma^{2}}\right), x>0,
$$

where $\mu$ is the scale parameter and $\sigma>0$ is the shape parameter, the gamma distribution with the shape parameter $\nu>1$ and the scale parameter $\beta>0$,

$$
f(x ; \nu, \beta)=\frac{x^{\nu-1}}{\beta^{\nu} \Gamma(\nu)} \exp \left(-\frac{x}{\beta}\right), x>0,
$$

and the beta distribution (Hahn and Shapiro, 1994). However the beta is not an appropriate probability distribution for this study because its domain is limited to $0<x<1$. Thus, the Rayleigh, lognormal, and gamma distributions, which are always unimodal and right skewed, are candidates for the probability distribution of EEG envelopes in this study. To determine the most suitable distribution, we fitted these three candidates to the estimated 100-bin histogram of $E(t)$. Then, the goodness-of-fit for each of these distributions was tested by evaluating the maximum log-likelihood. Moreover, in order to estimate and compare the sum of squares of the absolute difference between the empirical and candidate distributions, we introduced a standard least-square criterion

$$
\xi=\frac{\sqrt{\sum_{i=1}^{N_{b}} \delta_{i}^{2}}}{\left|p_{0.99}-p_{0.01}\right|},
$$

where the denominator is a normalization factor that is expressed in terms of the 99 th and 1 st percentiles for the distributions, $p_{0.99}$ and $p_{0.01}$, respectively, and the number of bins, $N_{b}=100$. An over $95 \%$ intercriteria agreement between the maximum log-likelihood and $\xi$ was achieved with a correlation of -1 , and, therefore, the parameter $\xi$ can be considered an intuitive and reliable index.

\subsection{Estimation of the statistics of the probability distribution}

To understand the stationary characteristics of the probability distribution, it is effective to estimate the statistics (mean, variance, mode, and skewness) of the distributions. For the Rayleigh of Eq. (3), the mean is defined as $\theta \sqrt{\frac{\pi}{2}}$, variance is $\left(2-\frac{\pi}{2}\right) \theta^{2}$, mode is $\theta$, and skewness is $\frac{2(\pi-3) \sqrt{\pi}}{(4-\pi)^{3 / 2}} \sim 0.63$. For the lognormal of Eq. (4), the mean is $\exp \left(\mu+\frac{\sigma^{2}}{2}\right)$, variance is $\exp \left(2 \mu+2 \sigma^{2}\right)-$ $\exp \left(2 \mu+\sigma^{2}\right)$, mode is $\exp \left(\mu-\sigma^{2}\right)$, and skewness is $\left(\exp \left(\sigma^{2}\right)+2\right) \sqrt{\exp \left(\sigma^{2}\right)-1}$. For the gamma of Eq. (5), the mean is $\nu \beta$, variance is $\nu \beta^{2}$, mode is $(\nu-1) \beta$, and skewness is $\frac{2}{\sqrt{\nu}}$. Generally, the statistics are completely estimated once all of the maximum likelihood estimators (MLEs), $\hat{\theta}, \hat{\mu}, \hat{\sigma}, \hat{\nu}$, and $\hat{\beta}$, are known. Our above-described analytic procedures are summarized in Figure 1.

Place Figure 1 around here 


\section{Results}

Place Figure 2 and Table 1 around here

Figure $2 \mathbf{a}$ shows the result of the estimation of the percentage (\%) of low-voltage activities at the $\mathrm{C} 3$ electrode. It shows that the percentage decreased uniformly as PCA increased. In the regression analysis with PCA as an independent variable and percentage as the dependent variable, we found that the two variables had a linear relationship, $y=b_{0}+b_{1} \times$ PCA. Here, $b_{0}$ was a constant, while $b_{1}$ was the regression coefficient. At the C3 electrode, $b_{0}=1.53, b_{1}=-0.03$, and $R^{2}=0.73(p<0.01)$. For the other five electrodes (Fp1, Fp2, C4, O1, and O2), we obtained similar relationships, and the details are summarized in Table 1. As the relationships between PCA and the two remainders (maximum and mean duration of the low-voltage activities) were not linear, a logarithmic transform was applied to the data of maximum and mean. The maximum at the C3 electrode decreased exponentially with PCA $\left(\log _{10}(\right.$ Maximum $)=2.69-0.04 \times$ PCA; $\left.R^{2}=0.57 ; p<0.01\right)$ as shown by the triangular symbols in Figure $2 \mathbf{b}$. The mean at the C3 electrode exponentially decreased with PCA $\left(\log _{10}(\right.$ Mean $)$ $\left.=1.62-0.02 \times \mathrm{PCA} ; R^{2}=0.60 ; p<0.01\right)$ as shown by the circular symbols in Figure 2b. The details of the results for both the maximum and mean are summarized in Table 1.

Place Figure 3 and 4 around here

Figure 3 shows two representative histograms of the envelope and three probability distributions that were estimated numerically with MLEs. The results of estimating the $\xi$ values for the three distributions are shown in Figure 4. It should be noted that the $\xi$ values for the gamma distribution decreased exponentially as PCA increased, and at 44 weeks PCA, the $\xi$ values for the distribution reached their minimum value. We classified the preterm EEGs based on the infants' PCAs: 10 infants at 38 weeks or less, and 10 infants at greater than 38 weeks, and we found a significant difference between them. Namely, at 38 weeks PCA or less, $98 \%(n=59)$ of the $\xi$ values for the lognormal distribution were estimated as small values, while the remaining $(2 \%)$ showed a gamma distribution. This indicated that the lognormal distribution provided a suitable fit model for the EEG envelopes in the preterm period. However, at greater than 38 weeks PCA, a lognormal distribution was identified in only 45 envelopes $(75 \%)$. The remaining 15 envelopes (25\%) showed a gamma distribution. Then, we used Fisher's exact test to determine the significance of the difference between the two groups. As a result, there was a statistically significant difference between the two groups $(p<0.01)$. This indicated that the suitable probability distribution for the envelopes changed from lognormal to gamma in the neonatal period, and, thus, gamma may provide a better model at the end of the neonatal period. 
This result also suggested that the stationary characteristics of the envelope for preterm EEGs gradually changed during the neonatal period.

Place Figure 5 and Table 2 around here

As shown in Figure 5a, significant positive correlations were found between the MLE $\hat{\mu}$ and PCA at the C3 electrode. The Pearson product-moment correlation coefficients $r$ were $0.5-0.7(p<0.01)$ in all six electrodes, as listed in Table 2. Similarly, the reciprocal of the MLE $\hat{\sigma} ;$ i.e., $(\hat{\sigma})^{-1}$, showed significant positive correlations at the $\mathrm{C} 3$ electrode (Figure $5 \mathbf{b}$ ). For both lognormal parameters, increasing linear trends with PCA were observed in all six electrodes. It was remarkable that an extremely strong negative correlation between $\hat{\mu}$ and the percentage of discontinuity was observed at the C3 electrode (Figure 5c). We then calculated $r$ between the $\hat{\mu}$ and the percentages, and the results was -0.96. At the Fp1, Fp2, C4, and O1 electrodes, $r$ were $-0.97,-0.94,-0.97$, and -0.97 , respectively. The $r$ at $\mathrm{O} 2$ was -0.87 . These facts indicated that $\hat{\mu}$ becomes an index that is inversely proportional to discontinuity.

Place Table 3 around here

Columns 2, 4, and 6 of Table 3 summarize the statistics (mean, variance, and skewness) for the probability distribution that was estimated with the MLEs for a representative case with a PCA of 32 weeks. When looking only at the values of the mean, the difference between the lognormal and gamma distribution was not clear. However, the skewness was clearly different in the lognormal, 6.64, and gamma distribution, 1.89. As shown in Figure 3a, in preterm EEGs of a case with a PCA of 32 weeks, the lognormal was appropriate due to the remarkably skewed shape of the probability distribution. However, the situation changed as PCA increased. In the case with a PCA of 44 weeks that is shown in Figure $3 \mathbf{b}$, the asymmetric shape of the probability distribution was lost, which thus made the gamma distribution more appropriate. The estimated statistics are summarized in columns 3,5 , and 7 of Table 3 . This indicated that the asymmetry of the probability distribution gradually changed as PCA increased and suggested that the feature would be effective for understanding the inherent mechanisms in early human brain development.

Place Figure 6 and Table 4 around here

Figure 6 shows a positive correlation between the estimated mode and PCA. Simply put, the PCA increased linearly as the mode increased. We then performed a regression analysis with mode as an independent variable and PCA as 
the dependent variable. We found that the relationship between the two variables was a linear expression, PCA $=24.4+1.82 \times$ mode at the $\mathrm{C} 3$ electrode $\left(R^{2}=0.85, p<0.01\right)$. Similar relationships were obtained for the other five electrodes, and the details are shown in Table 4. These results indicated that the mode may be a useful index for PCA prediction.

\section{Discussion}

The purpose of this study was to determine the three stationary characteristics of discontinuity, asymmetry, and unimodality based on statistical mathematical analyses of the preterm EEG envelope. Our findings are summarized in the following statements. First, we determined the discontinuity in preterm EEGs using the percentage (\%), maximum, and mean duration of low-voltage activities. In particular, the percentages showed a negative linear relationship with PCA for all of the electrodes. The regression analysis results showed that the percentage of low-voltage activities decreased linearly: approximately $50 \%$ (range: $41 \%$ to $57 \%$ ) at 32 weeks PCA and approximately 20\% (range: $13 \%$ to $28 \%$ ) at 40 weeks PCA. However, the maximum duration showed an exponential decrease: approximately $40 \mathrm{~s}$ (range: $31 \mathrm{~s}$ to $52 \mathrm{~s}$ ) at 32 weeks PCA and approximately $20 \mathrm{~s}$ (range: $14 \mathrm{~s}$ to $27 \mathrm{~s}$ ) at 40 weeks PCA. In addition the mean duration showed an exponential decrease: approximately $11 \mathrm{~s}$ (range: $10 \mathrm{~s}$ to 13 s) at 32 weeks PCA and approximately $8 \mathrm{~s}$ (range: $7 \mathrm{~s}$ to $8 \mathrm{~s}$ ) at 40 weeks PCA. Thus, in the preterm EEGs, the discontinuous waveforms decreased uniformly as PCA increased. Although this decreasing tendency was consistent with the findings of other studies (Benda et al.,1989; Selton et al., 2000; Clancy et al., 2003; Conde et al., 2005; Niemarkt et al., 2010), the estimated values for the maximum and mean duration in this study were slightly longer than those of other studies. This may have been because the fact that our results were based not on visual inspection, but on numerical assessments of the envelope.

The remarkable result was that the MLE $\hat{\mu}$ of the lognormal distribution was found to have a positive correlation with PCA as well as a strong negative correlation with the percentage of low-voltage activity. Basically, the amplitude of preterm EEGs decreased with maturation, and the discontinuous pattern was shortened as the gestational ages of the infants increased (Anderson et al.,1985; Selton et al., 2000; Clancy et al., 2003; Vecchierini et al., 2007). Thus, the scale parameter of the lognormal $\mu$ was an index closely associated with the stationary characteristics of discontinuity, and estimating $\mu$ can reveal the developmental progress related to discontinuity (continuity) in preterm EEGs. These findings suggested that the plot before 31-33 weeks PCA that is seen in Figure 5a may describe the non-stationary nature of preterm EEGs due to the lack of continuous patterns.

Next, we identified the probability distribution of the preterm EEG envelopes with the maximum likelihood method. No envelope was identified as fitted by

the Rayleigh distribution. The reasons that the Rayleigh was not selected as the maximum likelihood model were the following. Because the Rayleigh is defined 
with only one parameter $\theta$, it has less flexibility to change the shape of the distribution. In practice, the skewness of a Rayleigh is always given by a constant value of 0.63 . In other words, the asymmetric feature of a Rayleigh is always constant. We showed that the estimated values of skewness decreased as PCA increased in the preterm EEGs. Therefore, the Rayleigh distribution was not appropriate for this study. Another reason was that the Rayleigh is interpreted as the sum of the stochastic variables that follow a normal distribution. In our study, if we considered the preterm EEG $x(t)$ and its Hilbert transformation $H(x(t))$ and assumed their normality, the envelope may have fit well to the Rayleigh. However, our results revealed that the normality assumption for the preterm EEGs could not be satisfied. This indicated that the NBGR process was inappropriate as a model for describing the envelope statistics of preterm EEGs. Because the EEGs showed varied phenomena including abrupt transitions between low-voltage activity and large-amplitude burst-like patterns along with continuous and discontinuous patterns, the intrinsic dynamic features of the envelope appeared to be essentially different from those described by the NBGR.

The probability distributions for the envelope of preterm EEGs were best fitted by lognormal distributions at 38 weeks PCA or less, and by gamma distributions at 44 weeks PCA. It was notable that we observed a role for skewness throughout the distribution transition. Namely, the transition from the lognormal to gamma arose with the decrease in the skewness of the distribution. Note that the skewness can be defined by using only the shape parameter $\sigma$ in a lognormal. In addition, we showed significant positive correlations between $(\hat{\sigma})^{-1}$ and PCA in all six electrodes. We indicated that the asymmetric characteristics of the probability distribution gradually decreased until about 44 weeks PCA. Thus, the parameter $(\hat{\sigma})^{-1}$ can be interpreted as an index that mainly reflects the asymmetric features of the distribution, such as the amount of irregular waveforms with high-voltage amplitudes. However, $(\sigma)^{-1}$ is known as an index of regularity for interstimulus interval distributions (Shimokawa and Shinomoto, 2009). Therefore, our findings in terms of the $(\hat{\sigma})^{-1}$ of asymmetry indicated that the temporal variation of the envelope progressively changed from a burst to a more rhythmic pattern as PCA increased. However, as shown in Figure $5 \mathbf{b}$, the plots of $(\hat{\sigma})^{-1}$ were unstable before $31-33$ weeks PCA. This suggested that the asymmetric features of preterm EEGs present a steadily increase throughout the latter half of the last trimester.

The findings concerning asymmetry may have clinical implications. Recently, amplitude-integrated EEG (aEEG) has become commonly used as a brain function monitor for infants in neonatal intensive care units (Hellström-Westas et al., 2008; Toet and Lemmers, 2009) since aEEG gives a compressed expression of the variation of EEG amplitude and enables an evaluation of the trends in electrocortical activity by simple pattern recognition. Sisman et al. (2005) have reported that, for infants with very low PCAs, a parameter of aEEG tracings called the lower border is below $5 \mu \mathrm{V}$. Burdjalov et al. (2003) have reported that the lower border gradually increases as PCA increases. Consequently, the interval between the lower and upper parameters of aEEG narrows 
with increasing PCA. Therefore, aEEG can be used clinically as a predictor of neurodevelopmental outcomes, but, until now, no study has explained the reason for the usefulness of aEEG mathematically. Here, we showed that the indications could be well understood by examining the statistical properties of the envelope. It is obvious that the probability distribution of the envelope for infants with very low PCAs is reasonably described by the lognormal law and that the parameter $(\sigma)^{-1}$ increases with PCA, as shown in Figure $4 \mathbf{b}$. Let us consider the third-moments of the lognormal distribution, namely the skewness as $\left(\exp \left(\sigma^{2}\right)+2\right) \sqrt{\exp \left(\sigma^{2}\right)-1}$. The decreasing trends in $(\sigma)^{-1}$ do not change the form of the distribution, only the distribution skewness. Consequently, at very low PCA, the distribution is long-tailed and skewed to the right-hand side. However, as PCA increases, the distribution is positively scaled but less skewed. These observations clearly explain the clinical evidence, as described above.

The transition suggested that preterm EEG envelopes form two stochastic models. Namely, the stochastic processes of preterm EEG envelopes can be categorized as lognormal processes in the preterm period and gamma processes in the late neonatal period. From a mathematical standpoint, the difference between these two stochastic processes is thought to indicate the presence of a nonlinear drift term. Thus, the present study suggested that nonlinear feedback becomes gradually strong as PCA approaches 44 weeks. This is likely to be interpreted as functional and structural changes in the infants' brain. According to neurobiological studies on human fetal development (Kostovic and Jovanov-Milosevic, 2006; Kostovic and Judas,2007; Vanhatalo and Lauronen, 2006), the thalamocortical connections are just arriving at the subplate-cortex layers in the early preterm period before 28 weeks PCA, and the thalamocortical connections are established in the cortical layer, while the corticocortical connections are innervating their final target layers, in the neonatal period. The generation of EEG signals requires that sufficiently large cortical networks are activated through the thalamocortical or long-range corticocortical connections within the hemisphere (Judas et.al. 2005; Huppi and Dubois, 2006). These findings led us to speculate that the observed switching was related to the maturation of cortical networks or to the loss of the presumed subplate systems. However, the incidence of spontaneous activity transients (SATs) declines during the perinatal period and disappears by a few weeks after birth (Vanhatalo and Kaila, 2006). Vanhatalo et al. (2005) have noted that the decline in SATs parallels the transition in the effects of GABA from depolarizing to hyperpolarizing. These findings led us to speculate again that the switching to gamma may be related to the loss of the presumed subplate system and the changes in GABAergic neuron properties. Presently, it is not possible to elucidate an actual physiological explanation that describes such dynamic features. Such physical and physiological modeling of early human brain development with preterm EEGs is a topic to be addressed in future studies.

In addition, we proposed a new method for the clinical evaluation of brain maturation. Because the probability distribution of preterm EEG envelopes is always identified as lognormal or gamma, which are both unimodal, the mode appears to show a definite increasing trend with PCA. According to the results 
of the regression analysis, PCA can be estimated as approximately 35 weeks (range: 33.1 weeks to 35.7 weeks) if the mode equals 5.0 and approximately 44 weeks (range: 41.7 weeks to 46.3 weeks) if the mode equals 10.0. This indicates that it is possible to predict the infants' PCA with mode. In a lognormal distribution, the mode can be defined by $\exp \left(\mu-\sigma^{2}\right)$. This suggests that the mode is associated with both discontinuity as $\mu$ and asymmetry as $\sigma$. Given this, we can consider that the mode is related to a comprehensive feature, such as the ongoing (continuous, oscillatory, or regular) activity of preterm EEGs.

EEG maturational patterns for preterm infants have been reported at each specific PCA and during different sleep states. They are based on temporal EEG features, the amplitude and frequencies of background activities, as well as the occurrences of age-specific waveforms and patterns. Practically, sleep stages are indistinct in preterm infants of 31 weeks PCA or earlier. In addition, the question of when definite sleep states first appear has still not been fully answered, and the answer depends on the behavioral and physiological parameters that are selected to identify sleep states (Curzi-Dascalova et al., 2008). Moreover, we refrained from classifying sleep stages to maximize the robustness of the stationary characteristics of probability distributions. Thus, this study describes continuous developmental changes that are not based on sleep stage, but are from a comprehensive standpoint of overall sleep, and we therefore applied a statistical and mathematical analysis to the whole data set of preterm EEGs.

In relation to a given time series, the method for deriving the square root of two squared can be used as the most classical method to obtain information about the amplitude. The envelope that was obtained in this paper is also one of mathematical variables that contain information about the amplitude. However, they should be considered to reflect different characteristics. If the time series of EEG is considered as random noise as shown by Saunders (1963), the amplitude can be assumed to follow a normal distribution. Without the loss of generality, if it conforms to $\mathrm{N}(0,1)$, it is possible to drive that the square of a standard normal variable follows a chi-square distribution with one degree of freedom. Therefore the probability distribution by the classical approach should be identified as chi-square, half-normal, or other similar distribution. These are nothing but a J-shaped form distribution with the maximum value at 0 and a curve extending to the right hand side. Namely the distribution does not become unimodal in nature. Thus it is not possible to obtain the results of this paper if the classical approach was applied.

In general, however, determining the most suitable algorithm of envelope extraction for a given time series is an issue that has yet to be solved mathematically. Since the preterm EEGs are not considered as a monocomponent signal, the envelope extracted by Hilbert transform is strictly not the same as the true envelope (Boashash, 1992). Even if another algorithm is applied to extract an envelope, such as cubic spline interpolation algorithm, we had confirmed that there was no difference in the extraction accuracy and the identified types of probability distribution. The accuracy of the envelope extraction algorithm was considered to have little influence on the results within the scope of 
the present study. We analyzed EEGs with a sufficient data length: $\mathrm{N}>10^{6}$. For this reason, although deviation between the envelopes extracted from different algorithms certainly existed, that deviation's distribution was expected to follow a normal according to the central limit theorem. In addition, it was expected that the standard deviation of the distribution would decrease in inverse proportion to the square root of the data length. As a matter of fact, we judged that the algorithm of envelope extraction had no effect on identifying the probability distribution of envelope of preterm EEGs.

The main limitation of the present study was the relatively low number of subjects. We performed repeated recordings in four infants: one infant was recorded four times, two infants were recorded three times, and one infant was recorded twice. These recordings were performed at intervals of at least 1 week. Because the purpose of our analysis was not to distinguish transient waveforms that were superimposed onto preterm EEGs, we sought to identify the probability distributions from preterm EEG envelopes so that we could extract stationary characteristics that do not depend on individual differences. Therefore, the repeated recordings were considered to have little effect on our results. However, further studies that are conducted in large populations and that include longitudinal recordings and other behavioral and neurophysiological sleep state descriptions are required to confirm our data and to help us better understand early human brain development.

In conclusion, we performed a statistical mathematical analysis of the envelope that was extracted from preterm EEGs. It was shown that the probability distribution of preterm EEG envelopes could be described with a lognormal distribution before 38 weeks PCA and by a gamma distribution at 44 weeks PCA, and we observed a role for skewness throughout the distribution transition. Namely, the transition arose with the decrease in the skewness of the distribution. It was also shown that the scale parameter of the lognormal distribution, $\mu$, was an index closely associated with the stationary characteristics of discontinuity, that the skewness, defined solely by the shape parameter, could be interpreted as an index that mainly reflected asymmetric features, such as the amount of irregular waveforms which did not occur frequently and which deviated significantly from the mean amplitude, and that the mode that was defined by both the scale and shape parameters may be related to comprehensive features of preterm EEGs, such as the steady increase of ongoing (continuous, oscillatory, or regular) activity. Therefore, we suggest that statistics that are derived from the probability distribution can be considered useful indexes for estimating stationary nature in developing brain activity in preterm EEGs. Furthermore, our statistical mathematical analyses of the preterm EEG envelope may be informative with regard to helping to understand early brain development in prematurely preterm infants. 


\section{Acknowledgments}

This work was supported in part by the Core Research for Evolutional Science and Technology (CREST) from the Japan Science and Technology Agency and Casio Science Promotion Foundation to G.T.

\section{References}

Anderson CM, Torres F, Faoro A. The EEG of the premature. Electroencephalogr Clin Neurophysiol 1985; 60:95-105.

Benda GI, EngelRC,Zhang YP. Prolonged inactive phases during the discontinuous pattern of prematurity in the electroencephalogram of very-low birth weight infants. Electroencephalogr Clin Neurophysiol 1989; 72:189-197.

Boashash B. Estimating and interpreting the instantaneous frequency of a signal - Part 1: Fundamentals. Proc IEEE 1992; 80:520-538.

Burdjalov VF, Baumgart S, Spitzer AR. Cerebral function monitoring: a new scoring system for the evaluation of brain maturation in neonates. Pediatrics 2003; 112:855-861.

Casey BM, McIntire DD, Leveno KJ. The continuing value of the Apgar score for the assessment of newborn infants. New Engl J Med 2001; 344:467-471.

Clancy RR, Bergqvist AGC, Dlugos DJ. Neonatal electroencephalography. In: Ebersole JS, Pedley TA. eds. Current practice of clinical electroencephalography, 3rd edition. Philadelphia: Lippincott Williams \& Wilkins, 2003:160-245.

Conde JR, Hoyos AL, Martinez ED, Campo CG, Perez AM, Hernandez Borges AA. Extrauterine life duration and ontogenic EEG parameters in preterm newborns with and without major ultrasound brain lesions. Clin Neurophysol 2005; 116: 2796-2809.

Connel JA, Oozeer R, Dubowitz V. Continuous 4 channel EEG monitoring: a guide to interpretation, with normal values, in preterm infants. Neuropediatrics 1987; 18:138-145.

Curzi-Dascalova, Giganti, Salzarulo. Neurophysiological basic and behavior of early sleep development. In: Marcus CL, Carroll JL, Donnelly DF, Loughlin GM, eds. Sleep in Children: Development Changes in Sleep Patterns, New York: CRC Press, 2008: 1-38. 
Dick DE, Vaughn AO. Mathematical description and computer detection of alpha waves. Math Biosci 1970; 7:81-95.

Dreyfus-Brisac C. The electroencephalogram of the premature infant and full-term newborn: Normal and abnormal development of waking and sleeping patterns. In: Kellaway P, Peters I, eds. Neurological and electroencephalograhic correlative studies in infancy. New York: Grune \& Stratton, 1964:186-206.

Eyre JA, Nanei S, Wilkinson AR. Quantification of changes in normal neonatal EEGs with gestation from continuous five-day recordings. Dev Med Child Neurol 1988; 30:599-607.

Goto K, Wakayama K, Sonoda H, Ogata T. Sequential changes in electroencephalogram continuity in very premature infants. Electroencephalogr Clin Neurophysiol 1992; 82:197-202.

Hahn GJ, Sgapiro SS. Statistical Models in Engineering. New York, John Wiley \& Sons, 1994.

Hayakawa M, Okumura A, Hayakawa F, Watanabe K, Ohshiro M, Kato Y, et al. Background electroencephalographic (EEG) activities of very preterm infants born at less than 27 weeks gestation: a study on the degree of continuity. Arch Dis Child Fetal Neonatal Ed 2001; 84:F163-167.

Hellström-Westas L, de Varie LS, Rosén I. Atlas of amplitude-integrated EEGs in the newborn, UK, Informa Healthcare, 2008.

Hughes JR, Fino JJ, Gagnon L. Period of activity and quiescence in the premature EEG. Neuropediatrics 1983; 14:66-72.

Hughes JR, Fino JJ, Hart LA. Premature temporal theta (PT2). Electroencephalogr Clin Neurophysiol 1987; 67:7-15.

Hüppi PS, Dubois J. Diffusion tensor imaging of brain development. Semin Fetal Neonatal Med 2006; 11:489-497.

Judas M, Rados M, Jovanov-Milosevic N, Hrabac P, Stern-Padovan R, Kostovic I. Structural immunocytochemical and MR imaging properties of preiventricular crossroads of growing cortical pathways in preterm infants. Am J Neuroradiology 2005; 26:2671-2784.

Kellaway P, Crawley JW. A primer of electroencephalography of infants, section I \& II: Methodology and criteria of normality. Houston: The Baylor University College of Medicine, 1964. 
Kostovic̀, I, Jovanov-Milosevic̀, N. The development of cerebral connections during the first 20-45 weeks' gestation. Semin Fetal Neonatal Med 2006; 11:415-422.

Kostovic̀, I, Judas, M. Transiden patterns of cortical lamination during prenatal life: do they have implications for treatment?. Neurosci Biobehav R 2007; 31:1157-1168.

Langley RS. On various definitions of the envelope of a random process. J Sound Vib 1986; 105:503-512.

Liang H, Lukkarinen S, Hartimo I. Heart sound segmentation algorithm based on heart sound envelogram. Comput Cardiol 1997; 24:105-108.

Middleton D. An Introduction to Statistical Communication Theory. New York, McGraw-hill, 1960.

Niemarkt HJ, Andriessen P, Peters CHL, Pasman JW, Zimmermann LJ, Bambang Oetomo S. Quantitative analysis of maturational changes in EEG background activity in very preterm infants with a normal neurodevelopment at 1 year of age. Early Hum Dev 2010; 86:219-224.

Parmelee AH, Wenner WH, Akiyama Y, Schultz M, Stern E. Sleep states in premature infants. Dev Med Child Neurol 1967; 9:70-77.

Rice SO. Methematical analysis of random noise. Bell Sys Tech J $1945 ; 23: 46-156$.

Saunders MG. Amplitude probability density studies on alpha and alpha-like patterns. Electroencephalogr Clin Neurophysiol 1963; 15:761767.

Scher MS. Ontogeny of EEG-sleep from neonatal through infancy periods. Sleep Med 2008; 9:615-636.

Selton D, Andre M, Hascoët JM. Normal EEG in very premature infants: reference criteria. Clin Neurophysiol 2000; 11:2116-2124.

Sheen YT. An envelope analysis based on the resonance modes of the mechanical system for bearing defect diagnosis. Measurement 2010; 43:912-934.

Shimokawa T, Shinomoto S. Estimating instantaneous irregularity of neural firing. Neural Comput 2009; 21:1931-1951.

Sisman J, Campbell DE, Brion LP. Amplitude-integrated EEG in 
preterm infants: Maturation of background pattern and amplitude voltage with postmenstrual age and gestational age. J Perinatol 2005; 25:391-396.

Sovijarvi ARA, Malmberg LP, Charbonneau G, Vanderschoot J, Dalmasso F, Sacco C, Rossi M et al. Characteristics of breath sounds and adventitious respiratory sounds. Eur Respir Rev 2000; 10:591-596.

Tayfun M, Lo J. Nonlinear Effects on Wave Envelope and Phase. J Water Port Coast Ocean Eng 2000; 116:79-100.

Toet, MC, Lemmers, PMA. Brain monitoring in neonates. Early Hum Dev 2009; 85:77-84.

Vecchierini MF, André M, d'Allest AM. Normal EEG of premature infants born between 24 and 30 weeks gestational age: Terminology, definitions and maturation aspects. Neurophysiologie Clinique 2007; $37: 311-323$.

Vanhatalo S, Palva JM, Andersson S, Rivera C, Voipio J, Kaila K. Slow endogenous activity transients and developmental expression of $\mathrm{K}^{+}{ }_{-} \mathrm{Cl}^{-}$cotransporter 2 in the immature human cortex. Eur J Neurosci 2005; 22:2799-2804.

Vanhatalo S, Lauronen L. Neunatal SEP - back to bedside with basic science. Semin Fetal Neonatal Med 2006; 11:464-470.

Vanhatalo, S, Kaila, K. Development of neonatal EEG activity: from phenomenology to physiology. Semin Fetal Neonatal Med 2006; 11:471-478.

Willekens H, Dumermuth GD, Meith D. EEG spectral power and coherence analysis in healthy full-term neonates. Neuropediatrics 1984; 15:180-190. 
Table 1: Summary of the regression analysis for the three discontinuous properties of the percentage, maximum, and mean duration of the low-voltage activities of preterm EEG envelopes. Note that a logarithmic (base-10) transform was applied to the maximum and mean.

\begin{tabular}{|c|c|c|c|c|c|c|c|c|c|}
\hline & & & & & & & $\overline{\text { Regressi }}$ & in & lues \\
\hline & $R^{2}$ & & $b_{0}[\mathrm{CI}]$ & & $b_{1}[\mathrm{CI}]$ & & wk. [CI] & & $v k .[\mathrm{CI}]$ \\
\hline percentag & & & & & & & & & \\
\hline Fp1 & $0.68^{* *}$ & 1.54 & {$[1.13 ; 1.94]$} & -0.03 & {$[-0.04 ;-0.02]$} & 51 & {$[24 ; 79]$} & 26 & {$[-2 ; 53]$} \\
\hline Fp2 & $0.73^{* *}$ & 1.61 & {$[1.21 ; 2.01]$} & -0.04 & {$[-0.05 ;-0.03]$} & 49 & {$[22 ; 76]$} & 21 & {$[-6 ; 48]$} \\
\hline C3 & $0.73^{* *}$ & 1.53 & {$[1.16 ; 1.91]$} & -0.04 & {$[-0.05 ;-0.03]$} & 45 & {$[19 ; 70]$} & 17 & {$[-9 ; 43]$} \\
\hline $\mathrm{C} 4$ & $0.73^{* *}$ & 1.53 & {$[1.14 ; 1.93]$} & -0.04 & {$[-0.05 ;-0.03]$} & 41 & {$[15 ; 68]$} & 13 & {$[-13 ; 40]$} \\
\hline O1 & $0.70^{* *}$ & 1.75 & {$[1.30 ; 2.20]$} & -0.04 & {$[-0.05 ;-0.02]$} & 56 & {$[25 ; 87]$} & 27 & {$[-4 ; 57]$} \\
\hline $\mathrm{O} 2$ & $0.72^{* *}$ & 1.72 & {$[1.30 ; 2.13]$} & -0.04 & {$[-0.05 ;-0.03]$} & 57 & {$[28 ; 85]$} & 28 & {$[0 ; 56]$} \\
\hline maximun & & & & & & & & & \\
\hline Fp1 & $0.49^{* *}$ & 2.61 & {$[2.01 ; 3.20]$} & -0.03 & {$[-0.05 ;-0.02]$} & 39 & {$[15 ; 98]$} & 22 & {$[8 ; 53]$} \\
\hline Fp2 & $0.58^{* *}$ & 2.81 & {$[2.20 ; 3.43]$} & -0.04 & {$[-0.06 ;-0.02]$} & 37 & {$[14 ; 97]$} & 18 & {$[7 ; 47]$} \\
\hline C3 & $0.57^{* *}$ & 2.69 & {$[2.10 ; 3.29]$} & -0.04 & {$[-0.05 ;-0.02]$} & 34 & {$[14 ; 88]$} & 17 & {$[7 ; 45]$} \\
\hline $\mathrm{C} 4$ & $0.60^{* *}$ & 2.81 & {$[2.18 ; 3.44]$} & -0.04 & {$[-0.06 ;-0.02]$} & 31 & {$[12 ; 84]$} & 14 & {$[6 ; 39]$} \\
\hline O1 & $0.44^{* *}$ & 2.75 & {$[2.04 ; 3.46]$} & -0.03 & {$[-0.05 ;-0.02]$} & 46 & {$[15 ; 140]$} & 25 & {$[8 ; 74]$} \\
\hline $\mathrm{O} 2$ & $0.34^{*}$ & 2.87 & {$[1.94 ; 3.80]$} & -0.04 & {$[-0.06 ;-0.01]$} & 52 & {$[12 ; 206]$} & 27 & {$[6 ; 114]$} \\
\hline mean & & & & & & & & & \\
\hline Fp1 & $0.69^{* *}$ & 1.66 & {$[1.42 ; 1.89]$} & -0.02 & {$[-0.03 ;-0.01]$} & 11 & {$[8 ; 16]$} & 8 & {$[6 ; 11]$} \\
\hline Fp2 & $0.63^{* *}$ & 1.70 & {$[1.41 ; 2.00]$} & -0.02 & {$[-0.03 ;-0.01]$} & 11 & {$[7 ; 17]$} & 7 & {$[5 ; 11]$} \\
\hline $\mathrm{C} 3$ & $0.60^{* *}$ & 1.62 & {$[1.34 ; 1.91]$} & -0.02 & {$[-0.03 ;-0.01]$} & 10 & {$[7 ; 16]$} & 7 & {$[5 ; 11]$} \\
\hline $\mathrm{C} 4$ & $0.50^{* *}$ & 1.56 & {$[1.24 ; 1.88]$} & -0.02 & {$[-0.03 ;-0.01]$} & 10 & {$[6 ; 17]$} & 7 & {$[5 ; 12]$} \\
\hline O1 & $0.54^{* *}$ & 1.70 & {$[1.37 ; 2.03]$} & -0.02 & {$[-0.03 ;-0.01]$} & 12 & {$[7 ; 19]$} & 8 & {$[5 ; 13]$} \\
\hline $\mathrm{O} 2$ & $0.52^{* *}$ & 1.80 & {$[1.41 ; 2.19]$} & -0.02 & {$[-0.03 ;-0.01]$} & 13 & {$[7 ; 23]$} & 8 & {$[5 ; 15]$} \\
\hline
\end{tabular}


Table 2: The estimated values of the Pearson's correlation coefficients for the two lognormal parameters $\mu$ and $(\sigma)^{-1}$ with PCA. For both of the parameters, significant positive correlations with PCA are shown in all six electrodes.

\begin{tabular}{ccc}
\hline electrode & $\hat{\mu}$ & $(\hat{\sigma})^{-1}$ \\
\hline Fp1 & $0.56^{*}$ & $0.61^{*}$ \\
Fp2 & $0.67^{*}$ & $0.63^{* *}$ \\
$\mathrm{C} 3$ & $0.54^{* *}$ & $0.55^{* *}$ \\
$\mathrm{C} 4$ & $0.64^{* *}$ & $0.52^{*}$ \\
$\mathrm{O} 1$ & $0.64^{* *}$ & $0.67^{* *}$ \\
$\mathrm{O} 2$ & $0.68^{* *}$ & $0.69^{* *}$ \\
\hline${ }^{*} p<0.05,{ }^{* *} p<0.01$.
\end{tabular}


Table 3: Comparison of the representative statistics estimated from the Rayleigh, lognormal, and gamma distributions. The values were estimated with the maximum likelihood estimators for infants with PCA 32 and 44 weeks.

\begin{tabular}{|c|c|c|c|c|c|c|}
\hline \multirow[b]{2}{*}{ PCA } & \multicolumn{2}{|c|}{ Rayleigh } & \multicolumn{2}{|c|}{ lognormal } & \multicolumn{2}{|c|}{ gamma } \\
\hline & $32 \mathrm{wk}$. & $44 \mathrm{wk}$. & $32 \mathrm{wk}$. & $44 \mathrm{wk}$. & 32 wk. & $44 \mathrm{wk}$. \\
\hline mean & 18.5 & 24.1 & 13.9 & 22.9 & 13.6 & 22.2 \\
\hline variance & 9.0 & 12.6 & 18.9 & 18.7 & 12.8 & 14.5 \\
\hline skewness & 0.63 & 0.63 & 6.64 & 2.97 & 1.89 & 1.29 \\
\hline
\end{tabular}


Table 4: Summary of linear regression analysis between mode and PCA. The modes were completely estimated using the analytic formula of $\exp \left(\hat{\mu}-\hat{\sigma}^{2}\right)$ for a lognormal distribution and $(\hat{\nu}-1) \hat{\beta}$ for a gamma distribution.

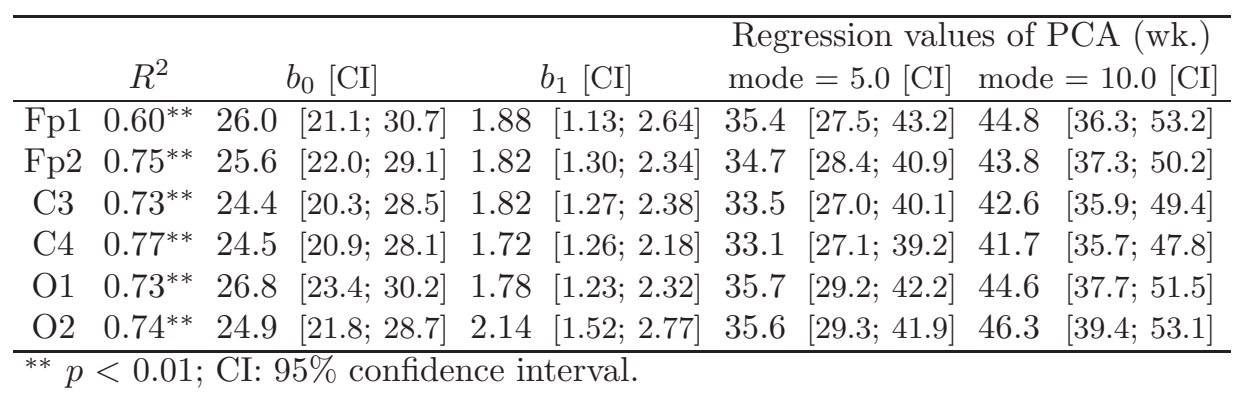


Figure 1: Schematic of EEG signal analysis conducted in this study. Preterm EEG signals were obtained from six electrodes (Fp1, Fp2, C3, C4, O1, and O2). Because the envelope provided a simpler and more conspicuous representation than that by raw data, we extracted it with the Hilbert transform. The discontinuity was evaluated by the percentage, the maximum duration, and the mean duration of the low-voltage activities $\leq 30 \mu \mathrm{V}$ and lasting for $>$ $5 \mathrm{~s}$. The histogram shows the frequency distribution of the extracted envelope. Then, the representative probability distribution of the envelope was chosen from among the Rayleigh, lognormal, and gamma distributions based on the statistical criteria of the maximum loglikelihood method. To investigate the stationary characteristics of the probability distribution, the statistics (mean, variance, mode, and skewness) were estimated.

Figure 2: Estimated results for the low-voltage activities obtained from the envelope of preterm EEGs at the C3 electrode. a. The percentage of low-voltage activity decreased uniformly as PCA increased $\left(R^{2}=0.73 ; p<0.01\right)$. b. The maximum of the low-voltage activity as shown by the triangular symbols decreased exponentially with PCA $\left(R^{2}=0.57 ; p<0.01\right)$. The mean duration as shown by the circular symbols decreased exponentially with PCA $\left(R^{2}=0.60\right.$; $p<0.01)$. Note that the $\mathrm{Y}$-axis is logarithmic.

Figure 3: Two representative histograms of the envelope and probability distributions (blue line profile: lognormal distribution; red: gamma; green: Rayleigh) for the preterm EEGs. a. An example of a probability distribution well fitted by a lognormal distribution. The data were obtained with the C3 electrode that was recorded from an infant whose PCA was 32 weeks, and the parameters estimated by using the MLEs were $\hat{\mu}=2.10$ and $(\hat{\sigma})^{-1}=0.98$ for the lognormal distribution, $\hat{\nu}=1.29$ and $\hat{\beta}=0.10$ for the gamma distribution, and $\hat{\theta}=13.7$ for the Rayleigh distribution. b. An example of a probability distribution well fitted by a gamma distribution. The data were obtained with the $\mathrm{C} 3$ electrode that was recorded from an infant whose PCA was 44 weeks, and the estimated parameters in this case were: $\hat{\mu}=2.87$, $(\hat{\sigma})^{-1}=1.41, \hat{\nu}=2.41, \hat{\beta}=0.11$, and $\hat{\theta}=19.2$. 
Figure 4: Estimated measures of $\xi$ for the three distributions (blue diamonds: lognormal distribution; red circles: gamma distribution; green asterisks: Rayleigh distribution) at the C3 electrodes. Although the $\xi$ values for the Rayleigh distribution showed a significant exponential decrease $\left(R^{2}=0.74, p<0.01\right)$ with PCA, they were estimated as larger than those for the lognormal and gamma distribution. Therefore, the Rayleigh distribution was considered a poor fit to the envelope. However, the $\xi$ values for the lognormal distribution were estimated as the smallest set of values at a PCA of 38 weeks or less. However, the $\xi$ values for the gamma distribution showed a significant exponential decrease $\left(R^{2}=0.65, p<0.01\right)$ with increased PCA, and, thereafter, they became the smallest set of values at 44 weeks PCA. No significant variation with PCA was observed for the lognormal distribution. These tendencies were also consistent with the corresponding signals that were obtained from the Fp1, Fp2, C4, O1 and O2 electrodes.

Figure 5: The representative results of the estimation of the parameters for the lognormal distribution. a. The scale parameter $\hat{\mu}$ showed a significant positive correlation with PCA, and the $\hat{\mu}$ values especially illustrate a linear increasing trend after 31 weeks PCA. This suggested that the continuous activity of the preterm EEGs increased linearly from around 31 weeks PCA. b. The shape parameter $(\hat{\sigma})^{-1}$ showed a significant positive correlation with PCA. Because the skewness was defined with only $(\sigma)$, the $(\hat{\sigma})^{-1}$ values could be interpreted as an index that mainly reflects the asymmetric characteristics of the distribution. c. A strong negative correlation between $\hat{\mu}$ and percentages of low-voltage activities was found $(r$ $=-0.96 ; p<0.01)$. Although the plots were obtained from the C3 electrode, similar plots and correlations were observed for the Fp1, Fp2, C4, O1, and O2 electrodes.

Figure 6: Positive correlation between the mode and PCA $\left(R^{2}=0.73 ; p<0.01\right)$. PCA had a relationship as PCA $=24.4+1.82 \times$ mode at the $\mathrm{C} 3$ electrode. The dashed line indicates the $95 \%$ confidence limits between mode and PCA. The mode is completely defined by $\exp \left(\mu-\sigma^{2}\right)$, and this suggests that it is associated with both discontinuity as $\mu$ and asymmetry as $\sigma$. We consider mode is an index that describes comprehensive features, such as developing ongoing activity in the preterm neonate. In addition, it may be a useful index in PCA prediction. Similar relationships were observed for the Fp1, Fp2, C4, O1, and O2 electrodes. 


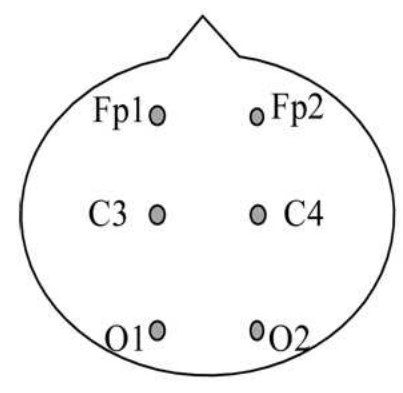

1. Preterm EEG Recording on 6 electrodes

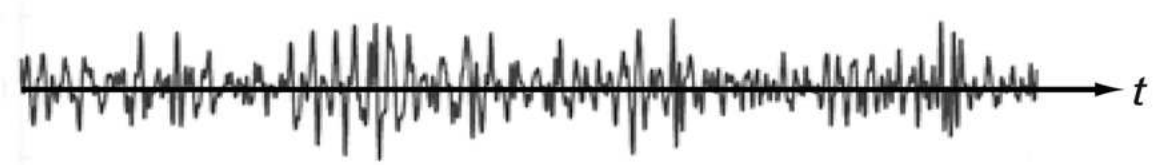

2. Envelope extraction using Hilbert transform

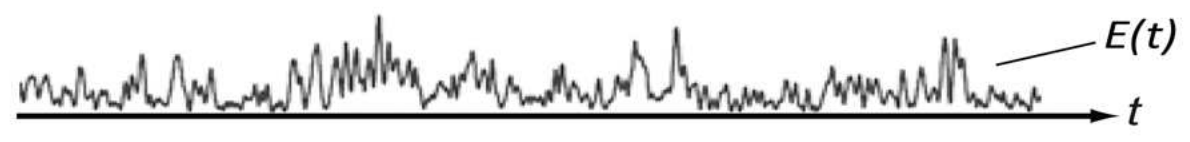

3. Evaluation of envelope characteristics

(i) Discontinuity

- Percentage of discontinuous patterns

- Maximun and mean duration of discontinuous patterns

(ii) Suitable probability distribution from three unimodals

- Rayleigh distribution
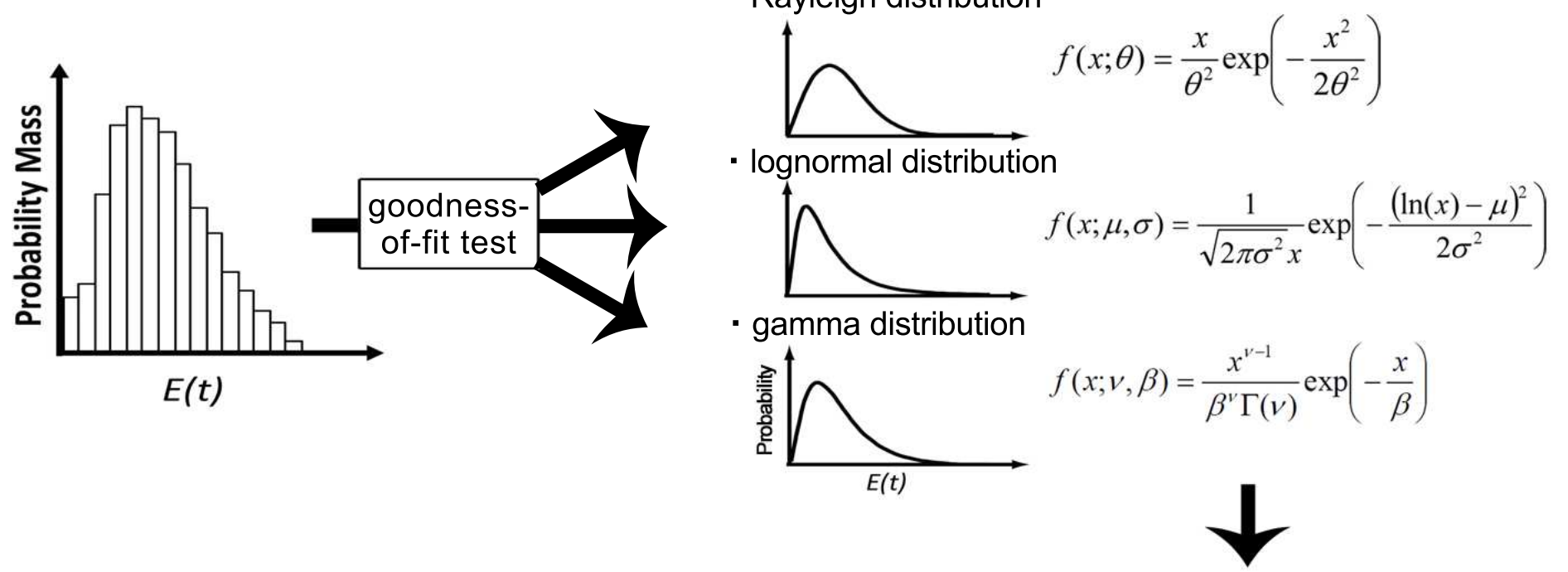

Statistical analysis 
a

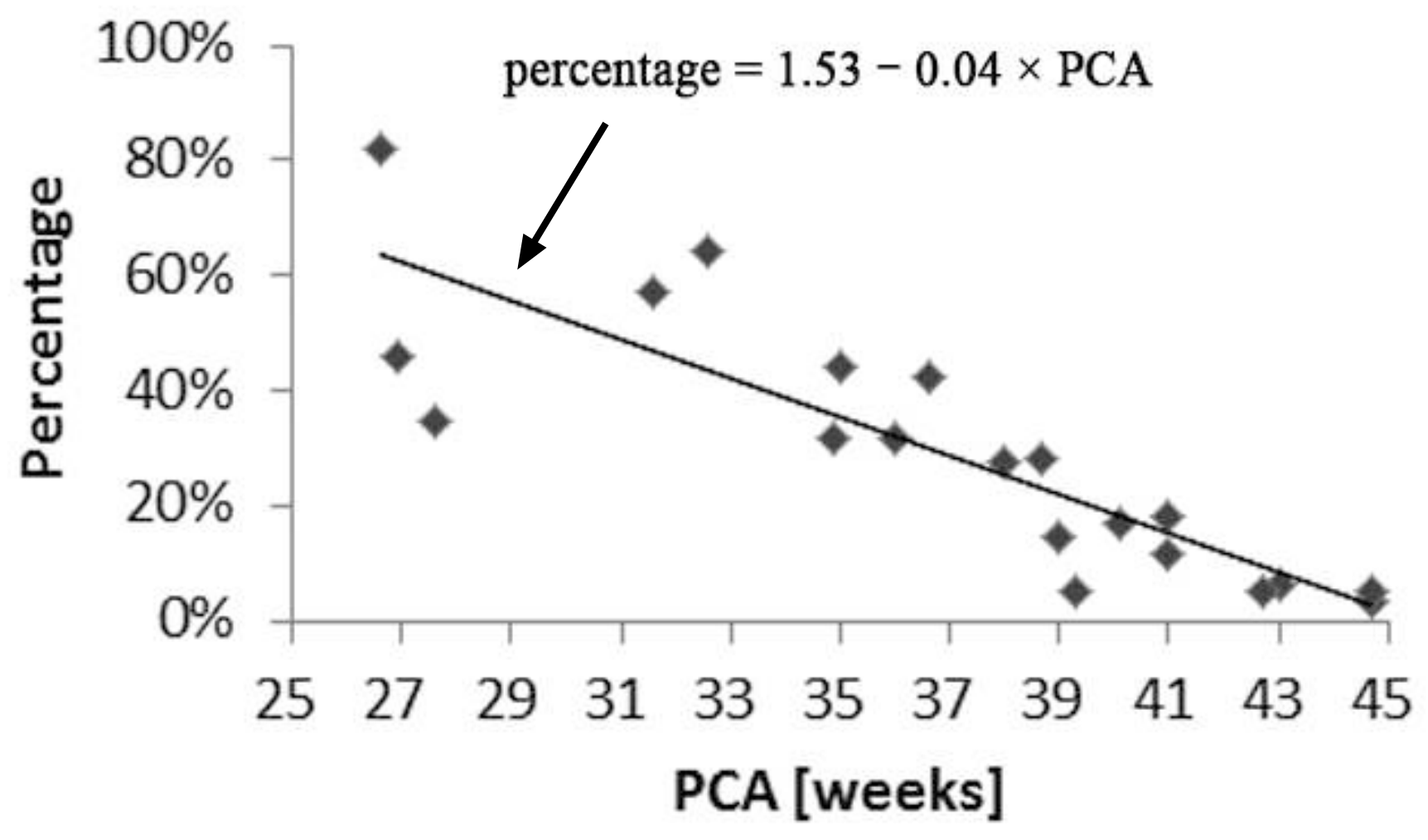

b

$\log _{10}(\max )=2.69-0.04 \times$ PCA

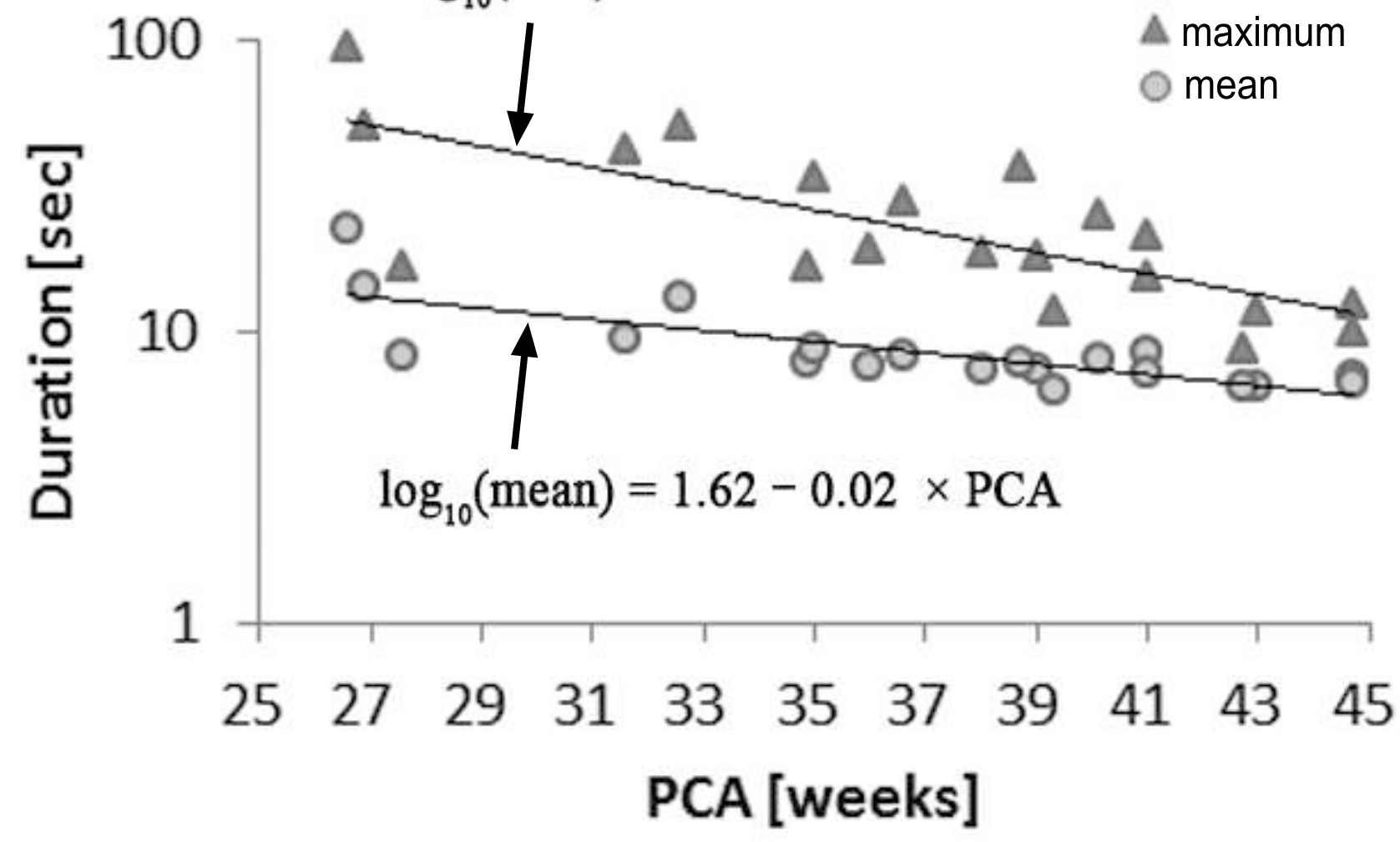


Figure3

a

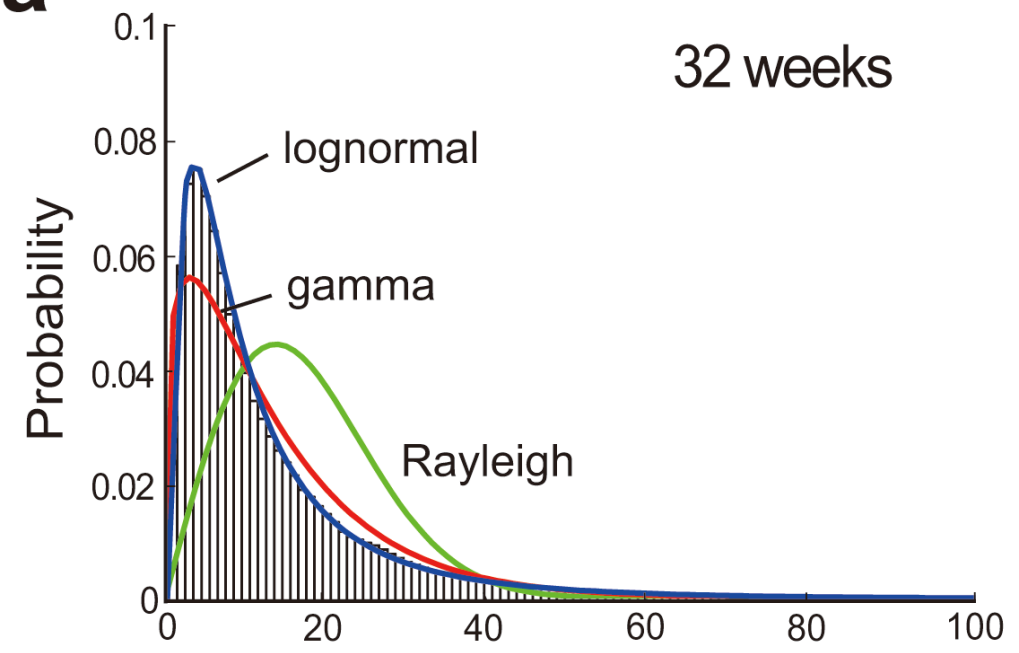

$E(t)[\mu \vee]$ b

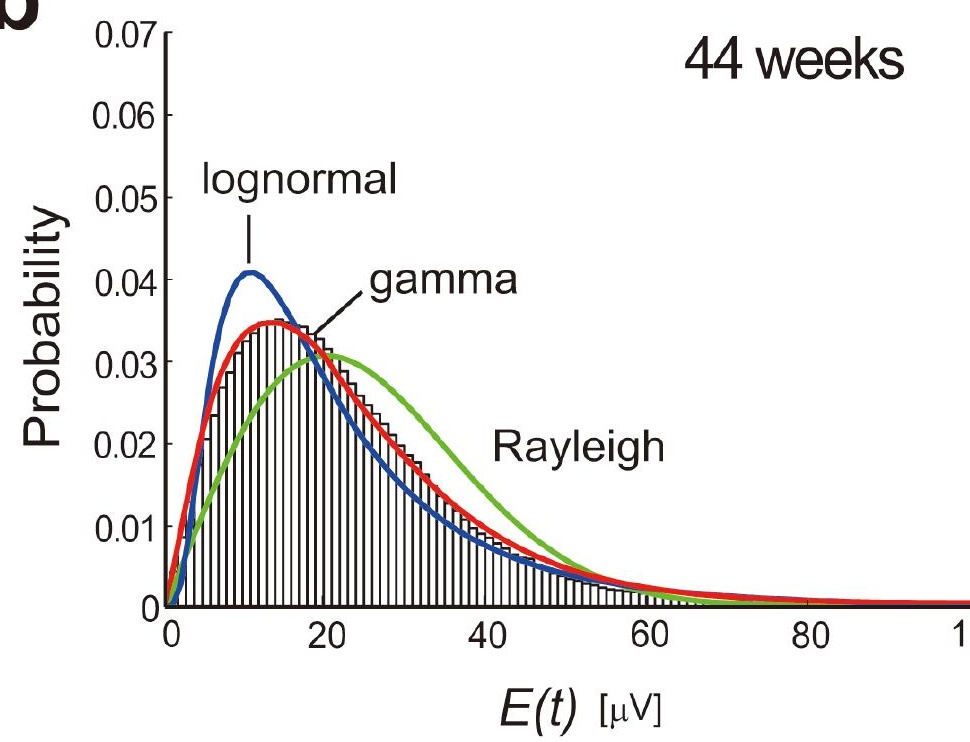


a

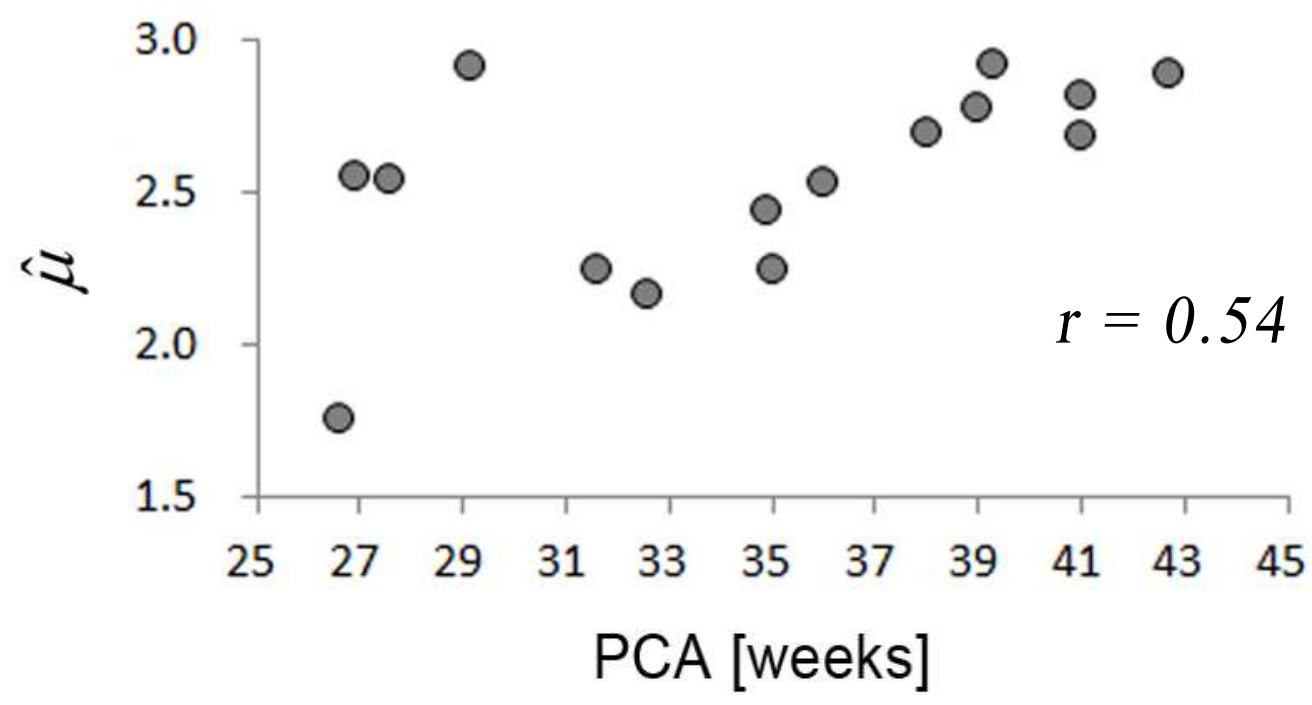

b

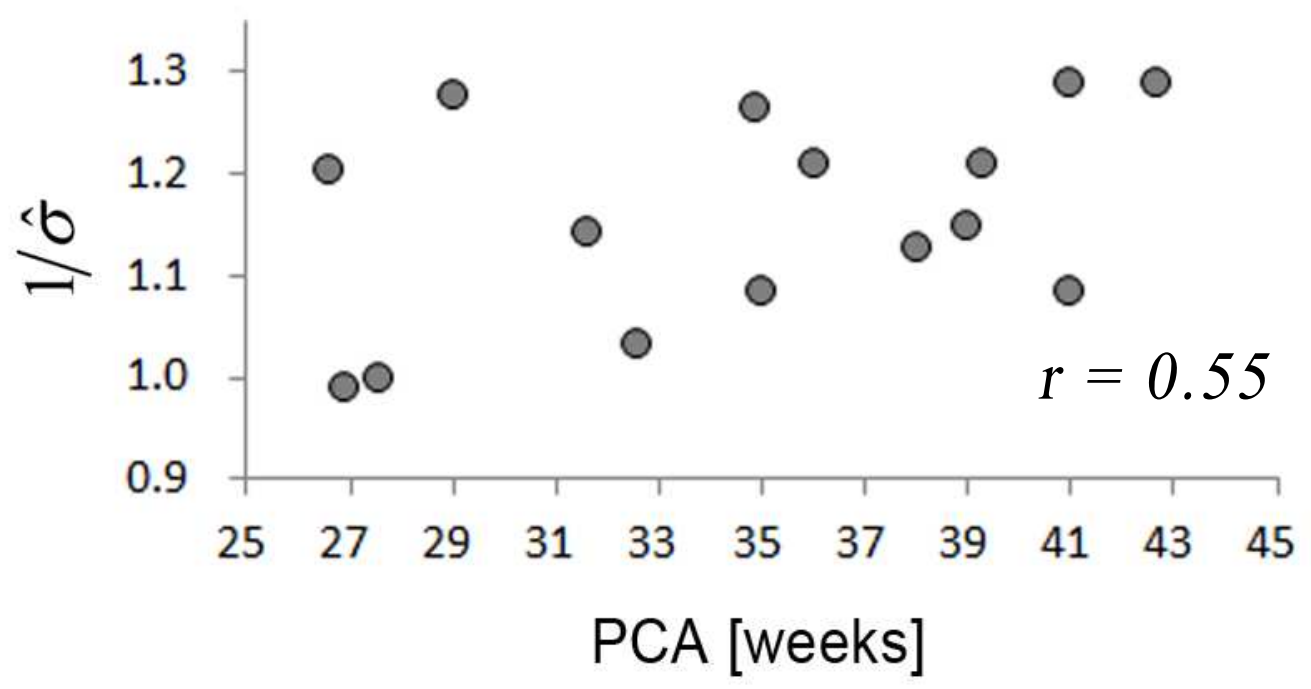

C

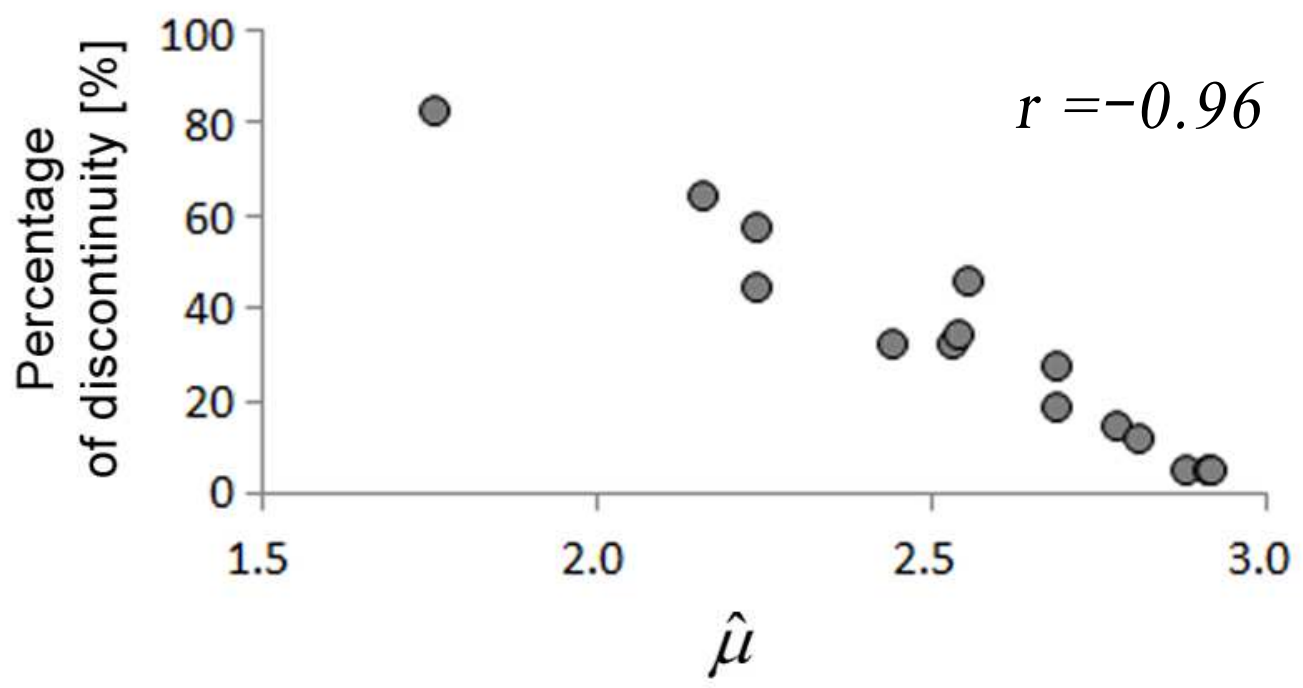




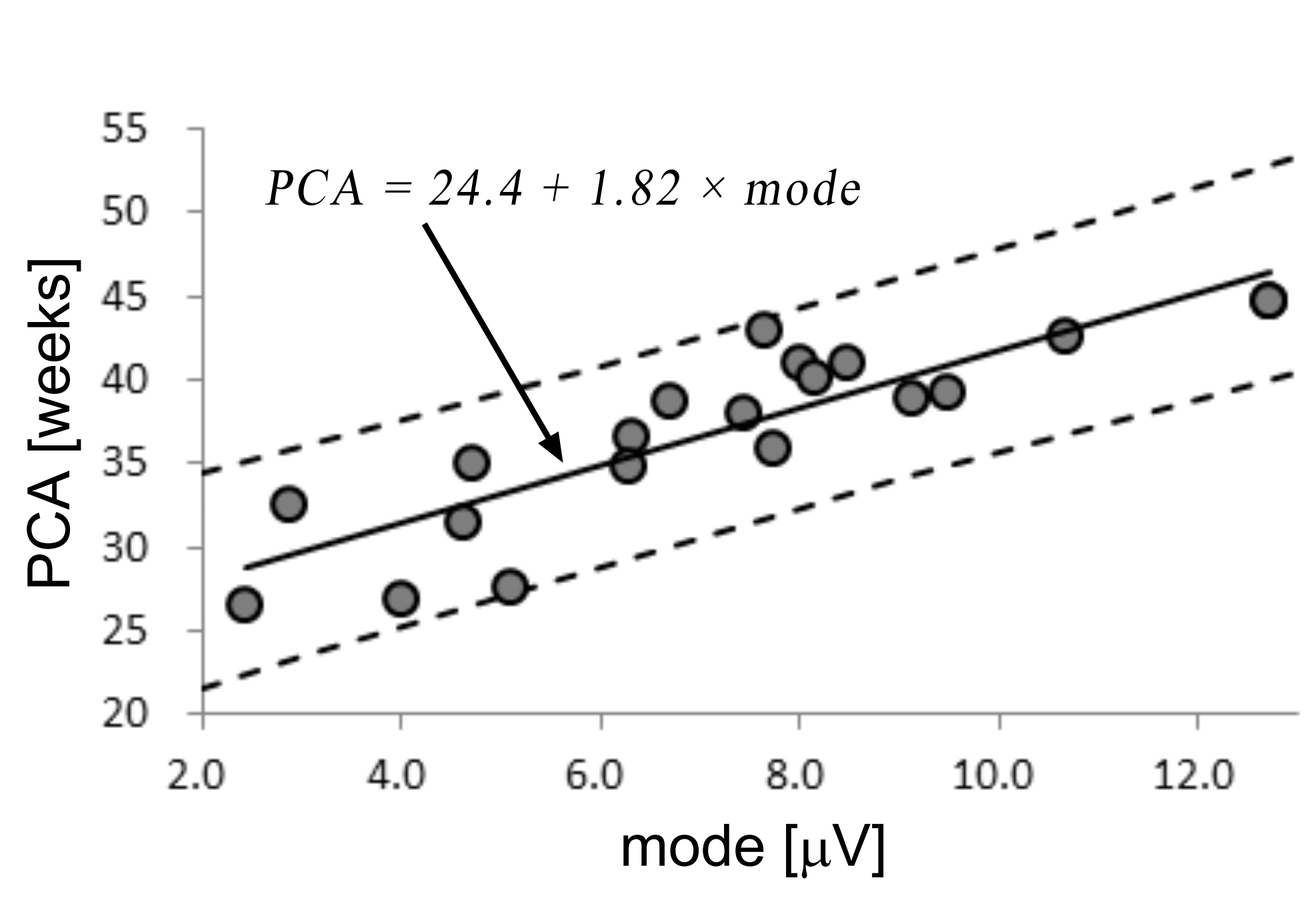

Figure6

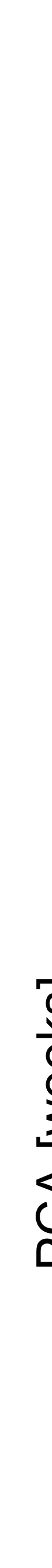

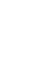
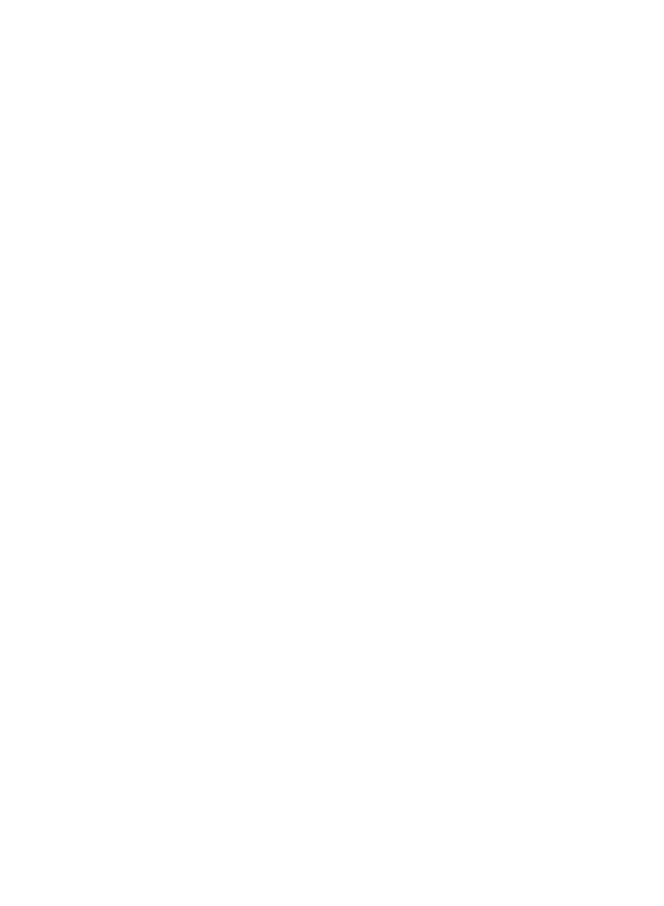

.
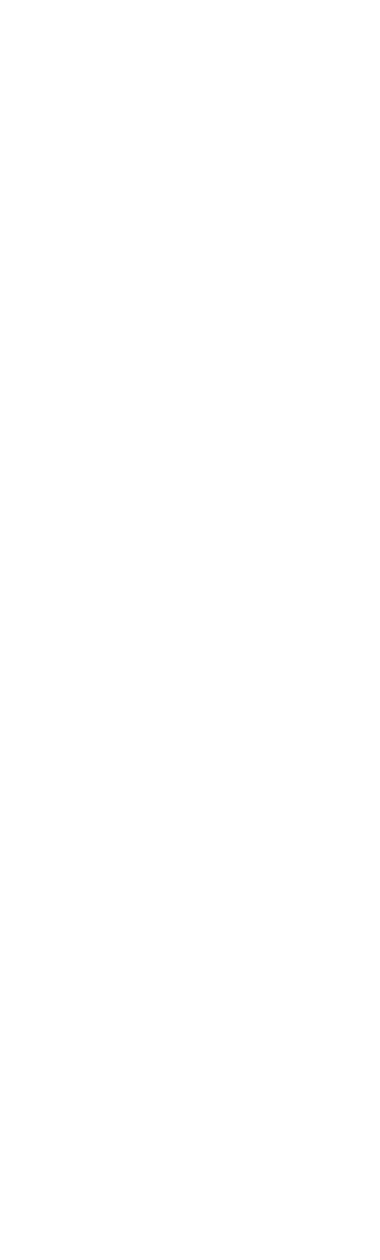

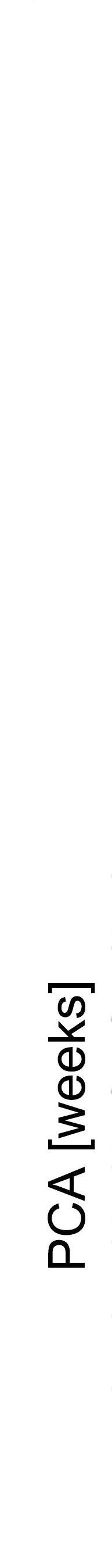

